\title{
Screening of long-range Coulomb interaction in graphene nanoribbons: Armchair versus zigzag edges
}

\author{
H. Hadipour, ${ }^{1, *}$ E. Şaşıŏlu ${ }^{2, \dagger}{ }^{\dagger}$ F. Bagherpour, ${ }^{1}$ C. Friedrich, ${ }^{3}$ S. Blügel,${ }^{3}$ and I. Mertig ${ }^{2}$ \\ ${ }^{1}$ Department of Physics, University of Guilan, 41335-1914 Rasht, Iran \\ ${ }^{2}$ Institute of Physics, Martin Luther University Halle-Wittenberg, 06120 Halle (Saale), Germany \\ ${ }^{3}$ Peter Grünberg Institut and Institute for Advanced Simulation, Forschungszentrum Jülich and JARA, 52425 Jülich, Germany
}

(Received 21 June 2018; revised manuscript received 1 October 2018; published 14 November 2018)

\begin{abstract}
We study the electronic screening of the long-range Coulomb interaction in graphene nanoribbons (GNRs) with armchair and zigzag edges as a function of the ribbon width by employing $a b$ initio calculations in conjunction with the random-phase approximation. We find that in GNRs with armchair edges quantum confinement effects lead to oscillatory behavior of the on-site screened Coulomb interaction with the ribbon width. Furthermore, the reduced dimensionality and the existence of a band gap result in a nonconventional screening of the Coulomb interaction; that is, it is screened at short distances and antiscreened at intermediate distances, and finally, it approaches the bare (unscreened) interaction at large distances. In the case of GNRs with zigzag edges the presence of edge states strongly affects the screening, which leads to a strong reduction of the effective on-site Coulomb interaction (Hubbard $U$ ) parameters at the edge. We find that the interactions turn out to be local; the nonlocal part is strongly screened due to edge states, making GNRs with zigzag edges correlated materials. On the basis of the calculated effective Coulomb interaction parameter $U$, we discuss the appearance of ferromagnetism at zigzag edges of GNRs within the Stoner model.
\end{abstract}

DOI: 10.1103/PhysRevB.98.205123

\section{INTRODUCTION}

The experimental synthesis of graphene has led to a huge growth in interest in the application of carbon-based materials for nanoelectronics [1-3]. Despite its extraordinary electronic and mechanical properties, graphene is not magnetic and has no band gap, which makes it difficult to use in practical digital circuits as well as for spintronic applications. Cutting graphene along particular directions, which produces graphene nanoribbons (GNRs) with armchair and zigzag edges, can change dramatically the electronic and magnetic properties of graphene [4-14]. GNRs with armchair edges are considered to be promising materials for low-power, high-frequency field-effect transistors due to their finite band gaps [15]. A number of spintronic devices based on spin-polarized edge states of GNRs with zigzag edges have been proposed [16-20]. Various fabrication techniques such as lithographic methods [14,21,22], unzipping of carbon nanotubes [23-26], and chemical synthesis [27-29] have been developed to produce high-quality GNRs with widths smaller than $10 \mathrm{~nm}$.

GNRs with armchair edges are classified into different categories depending on their electronic band structures $[6,7]$. The band gaps of 1-10-nm-wide armchair GNRs were found to be in the range 0.15 to $1 \mathrm{eV}$ in experiment [5,6], and they decrease with increasing ribbon width [5-7]. The reduced dimensionality in GNRs with armchair edges is expected to give rise to a significant enhancement of the Coulomb

\footnotetext{
*hanifhadipour@gmail.com

†ersoy.sasioglu@physik.uni-halle.de
}

interaction. The incorporation of electron-electron correlation effects within the $G W$ approximation results in large bandgap values of about $2.5 \mathrm{eV}$ in armchair GNRs of $1.5 \mathrm{~nm}$ width [30]. On the other hand, the interplay of reduced dimensionality and finite band gap results in nonconventional screening of the long-range part of the Coulomb interaction in low-dimensional semiconductors and insulators [31-35]. One of the consequences of such a nonconventional screening is the large exciton binding energy (as large as $1.5 \mathrm{eV}$ ) [36-41]. The latter was determined experimentally in armchair GNRs of $0.7-1-\mathrm{nm}$ width. This value is in good agreement with ab initio calculations [31,36,39]. Nonconventional screening of the Coulomb interactions occurs also in semiconducting carbon nanotubes [33]. In spite of several theoretical studies of Coulomb interactions in two-dimensional graphene [42-49] and the experimental indication of nonconventional screening in GNRs with armchair edges, up to now there has not been a study addressing Coulomb interactions from an ab initio perspective.

The existence of spin-polarized edge states in GNRs with zigzag edges makes these systems extremely important not only for technological applications but also for testing theories of magnetism for one-dimensional (quasi-one-dimensional) materials. According to the Mermin-Wagner theorem, a longrange magnetic order in one- and two-dimensional systems at finite temperatures is not possible [50]. However, theoretical studies have shown that due to the very high value of the spinwave stiffness $\left(D=2100 \mathrm{meV} \AA^{2}\right)$ the spin correlation length was found to be around $1 \mathrm{~nm}$ at room temperature [51,52]. Recent experiments provided evidence for ferromagnetism in GNRs with zigzag edges at room temperature [5]. The presence of edge states results in a relatively large contribution 
to the density of states (DOS) near the Fermi energy $E_{F}$, and thus even a weak Coulomb interaction makes the edge states magnetic within the Stoner model. The edge states are also expected to enhance the screening, and thus the local Coulomb interaction may change substantially across the GNRs. Moreover, the Coulomb interaction plays an important role in understanding electronic, magnetic, and transport properties of GNRs with zigzag edges [53].

In this paper we study the screening of the long-range Coulomb interaction between $p_{z}$ electrons in GNRs with armchair and zigzag edges as a function of ribbon width by employing $a b$ initio calculations in conjunction with the random-phase approximation (RPA) within the full-potential linearized augmented plane wave (FLAPW) method. We find that in GNRs with armchair edges the quantum confinement effects lead to oscillatory behavior of the on-site screened Coulomb interaction as a function of the ribbon width. Furthermore, the existence of the band gap and reduced dimensionality results in a nonconventional screening of the Coulomb interaction, which means that the interaction is screened at short distance, it is antiscreened at intermediate distances, and, finally, it is unscreened at large distances. Moreover, the obtained Coulomb interaction parameters in GNRs with armchair edges are larger than the ones in pristine graphene. In the case of GNRs with zigzag edges, the situation is quite different; the presence of edge states strongly affects the screening, which leads to a strong reduction of the effective Coulomb interaction (Hubbard $U$ ) parameter at the edges. The interactions turn out to be local; the nonlocal part is strongly screened due to edge states, making zigzag GNRs materials with much stronger local correlations than GNRs with armchair edges due to the larger gradient of the Coulomb interaction. On the basis of calculated effective Coulomb interaction parameters $U$ we discuss the appearance of ferromagnetism at zigzag edges of GNRs using the Stoner model. The rest of this paper is organized as follows. The method and computational details are presented in Sec. II. Section III deals with the results and discussion, and finally, in Sec. IV we give the conclusions.

\section{COMPUTATIONAL METHOD}

To consider two shapes of graphene nanoribbon edges, namely, zigzag and armchair edges, we use two different groups of conventional orthorhombic unit cells for simulations. In the first group [see Figs. 1(a)-1(d)], carbon atoms along the ribbon edges form an armchair pattern with the parameter $N_{a}$-GNRs. According to the convention in GNRs [6,7], armchair GNRs are classified into three categories: $N_{a}=3 p, N_{a}=3 p+1$, and $N_{a}=3 p+2$, where $N_{a}$ is the number of dimer lines across the ribbon width and $p$ is an integer. Here, in order to compare the systems in different categories we choose 6-AGNR, 7-AGNR, and 8-AGNR with the same $p=2$, and also we present the results for 9-AGNR to compare with 6-AGNR with the same category but different $p$. The second group, $N_{z}$-GNRs, with $N_{z}$ zigzag chains across the ribbon width, includes 6-ZGNR, 7-ZGNR, and 8-ZGNR, as depicted in Figs. 1(e)-1(g). We consider ribbon unit cells with a $25-\AA(15-\AA)$ vacuum separating them in the $z(y)$

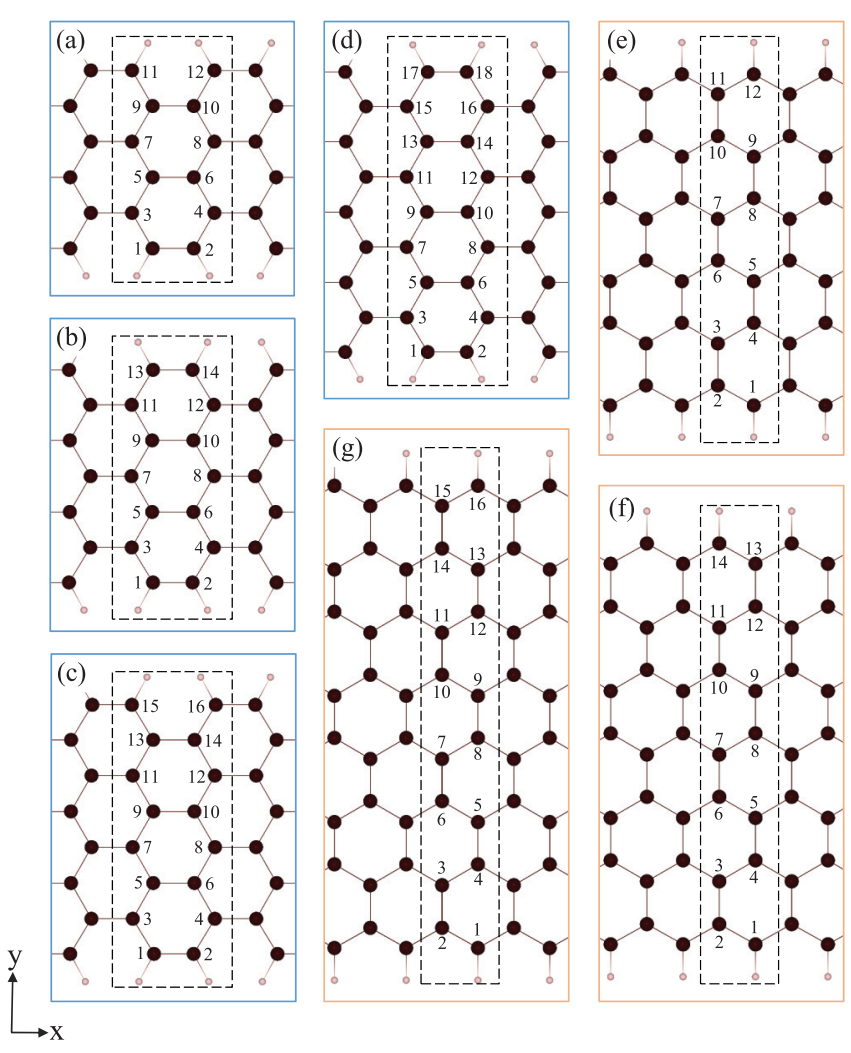

FIG. 1. Two types of edges for graphene nanoribbons: (a) $N_{a}=$ 6 (6-AGNR), (b) $N_{a}=7$ (7-AGNR), (c) $N_{a}=8$ (8-AGNR), and (d) $N_{a}=9$ (9-AGNR) are armchair ribbons with different widths. $N_{a}$ is the number of dimer lines across the ribbon width. (e), (f), and (g) correspond to zigzag ribbons of $N_{z}=6$ (6-ZGNR), $N_{z}=7$ (7ZGNR), and $N_{z}=8$ (8-ZGNR) respectively. $N_{z}$ is the number of zigzag chains across the ribbon width.

direction. The edge carbon atoms are passivated by hydrogen atoms. For comparison, pristine graphene is also considered.

In this work we study partially and fully screened Coulomb interaction parameters calculated with the ab initio constrained random-phase approximation (cRPA) and RPA methods, respectively. The fully screened Coulomb interaction $W$ is related to the bare Coulomb interaction $v$ as

$$
W\left(\boldsymbol{r}, \boldsymbol{r}^{\prime}, \omega\right)=\int d \boldsymbol{r}^{\prime \prime} \epsilon^{-1}\left(\boldsymbol{r}, \boldsymbol{r}^{\prime \prime}, \omega\right) v\left(\boldsymbol{r}^{\prime \prime}, \boldsymbol{r}^{\prime}\right),
$$

where $\epsilon\left(\boldsymbol{r}, \boldsymbol{r}^{\prime \prime}, \omega\right)$ is the dielectric function.

In the RPA of the dynamically screened Coulomb interaction, dielectric function is related to the electron polarizability $P$ by

$$
\epsilon\left(\boldsymbol{r}, \boldsymbol{r}^{\prime}, \omega\right)=\delta\left(\boldsymbol{r}-\boldsymbol{r}^{\prime}\right)-\int d \boldsymbol{r}^{\prime \prime} v\left(\boldsymbol{r}, \boldsymbol{r}^{\prime \prime}\right) P\left(\boldsymbol{r}^{\prime \prime}, \boldsymbol{r}^{\prime}, \omega\right),
$$

where the polarization function $P\left(\boldsymbol{r}^{\prime \prime}, \boldsymbol{r}^{\prime}, \omega\right)$ is given by

$$
\begin{aligned}
& P\left(\boldsymbol{r}, \boldsymbol{r}^{\prime}, \omega\right) \\
& =\sum_{\sigma} \sum_{\boldsymbol{k}, m}^{o c c} \sum_{\boldsymbol{k}^{\prime}, m^{\prime}}^{\text {unocc }} \varphi_{\boldsymbol{k} m}^{\sigma}(\boldsymbol{r}) \varphi_{\boldsymbol{k}^{\prime} m^{\prime}}^{\sigma *}(\boldsymbol{r}) \varphi_{\boldsymbol{k} m}^{\sigma *}\left(\boldsymbol{r}^{\prime}\right) \varphi_{\boldsymbol{k}^{\prime} m^{\prime}}^{\sigma}\left(\boldsymbol{r}^{\prime}\right) \\
& \quad \times\left[\frac{1}{\omega-\epsilon_{\boldsymbol{k}^{\prime} m^{\prime}}^{\sigma}+\epsilon_{\boldsymbol{k} m}^{\sigma}+i \eta}-\frac{1}{\omega+\epsilon_{\boldsymbol{k}^{\prime} m^{\prime}}^{\sigma}-\epsilon_{\boldsymbol{k} m}^{\sigma}-i \eta}\right] .
\end{aligned}
$$


Here, $\epsilon_{\boldsymbol{k} m}^{\sigma}$ are single-particle Kohn-Sham eigenvalues obtained from density functional theory (DFT), and $\eta$ is a positive infinitesimal. Further, $\varphi_{\boldsymbol{k} m}^{\sigma}(\boldsymbol{r})$ are the single-particle Kohn-Sham eigenstates with spin $\sigma$, wave number $\boldsymbol{k}$, and band index $m$. The tags occ and unocc above the summation symbol indicate that the summation is, respectively, over only occupied and unoccupied states.

The cRPA method allows us to calculate the effective Coulomb interaction (also called partially screened interaction) between $p_{z}$ electrons in GNRs. In this approach, in order to exclude the screening due to certain electrons we separate the full polarization function in Eq. (3) into two parts:

$$
P=P_{z}+P_{r},
$$

where $P_{z}$ includes only transitions between the $p_{z}$ states and $P_{r}$ is the remainder. Then, the frequency-dependent effective Coulomb interaction is given schematically by the matrix equation

$$
U(\omega)=\left[1-v P_{r}(\omega)\right]^{-1} v .
$$

Application of Eq. (4) to materials with entangled bands is not straightforward. Because $p_{z}$ states may be mixed with other extended $s, p_{x}$, and $p_{y}$ states at some parts of the Brillouin zone, there is no unique identification of the $p_{z} \rightarrow$ $p_{z}$ transitions for constructing $P_{z}$. To identify the correlated subspace and reveal the mixing of $p_{z}$ states with other bands in the GNRs, total and projected band structures for two systems, 7-AGNR and 6-ZGNR, are depicted in Figs. 2(a) and 2(b), respectively. The results for edge and inner $\mathrm{C}$ atoms are also reported separately. For both systems the contribution of $p_{z}$ states around $E_{F}$ is significant compared to $p_{x}, p_{y}$, and $s$ orbitals. The $p_{z}$ bands are disentangled from the rest in a large energy interval from -3 to $3 \mathrm{eV}$. For higher energies, $p_{z}$ states are crossed by other bands and also mixed with them, which means they are entangled bands. Several procedures have been proposed in the literature to calculate $P_{z}$ for entangled bands [54,55]. In this work we use the method described in Ref. [55]. Here, we first define the probability to find $p_{z}$ electrons of $\mathrm{C}$ atoms in eigenstate $\varphi_{\boldsymbol{k} m}^{\sigma}$ as

$$
c_{k m}^{\sigma}=\sum_{i, n}\left|T_{i, m n}^{\sigma k}\right|^{2}
$$

Here, the unitary matrices $T_{i, m n}^{\sigma k}$ are determined from the concept of maximally localized Wannier functions,

$$
w_{i n}^{\sigma}(\boldsymbol{r})=\frac{1}{N} \sum_{\boldsymbol{k}} e^{-i \boldsymbol{k} \cdot \boldsymbol{R}_{i}} \sum_{m} T_{i, m n}^{\sigma \boldsymbol{k}} \varphi_{\boldsymbol{k} m}^{\sigma}(\boldsymbol{r}),
$$

where $w_{i n}^{\sigma}(\boldsymbol{r})$ is a maximally localized Wannier function located at site $i, N$ is the number of discrete $k$ points in the full Brillouin zone, and $\boldsymbol{R}_{i}$ is the position vector of atomic site $i$. The matrices $T_{i, m n}^{\sigma k}$ are determined by minimizing the spread of the Wannier functions,

$$
\Omega=\sum_{i, n, \sigma}\left(\left\langle w_{i n}^{\sigma}\left|r^{2}\right| w_{i n}^{\sigma}\right\rangle-\left\langle\left. w_{i n}^{\sigma}|\boldsymbol{r}| w_{i n}^{\sigma}\right|^{2}\right) .\right.
$$

Here, the sum runs over all Wannier functions. From Eq. (7) it is clear that we need to know which bands should be included for the construction of the maximally localized Wannier states. Since $p_{z}$ bands are extended to very high
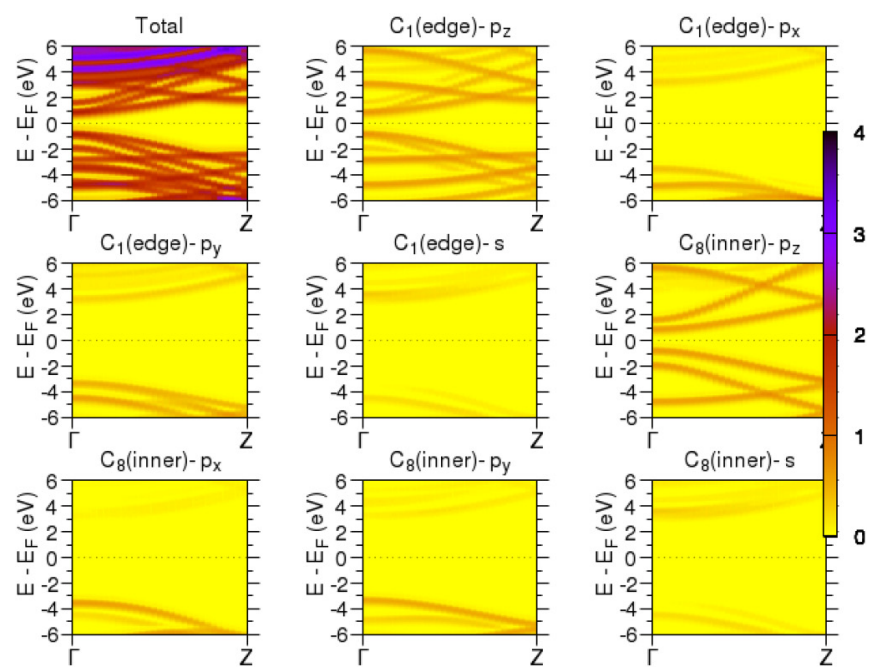

(a)
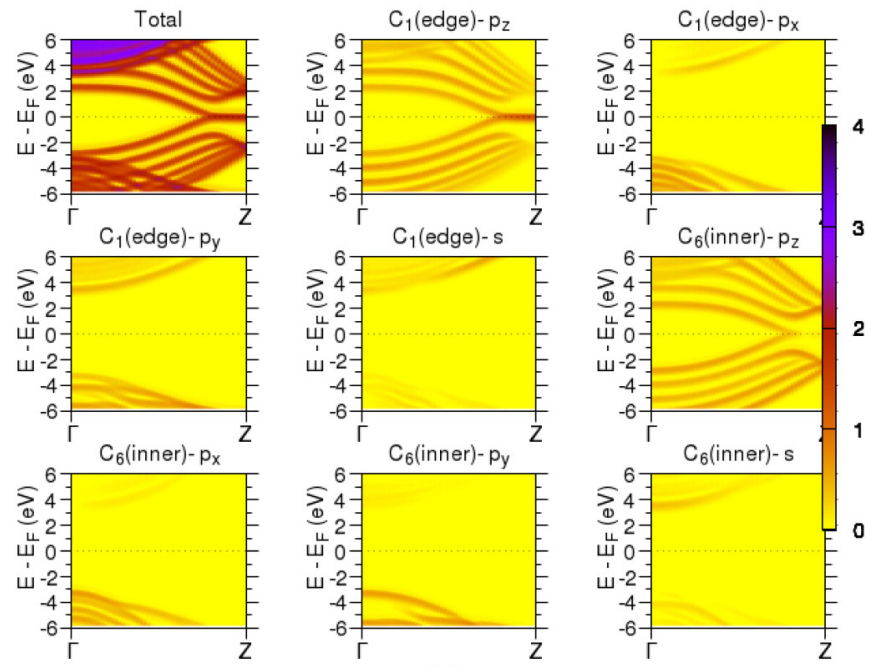

(b)

FIG. 2. (a) Total and orbital-projected band structures for edge and inner $\mathrm{C}$ atoms of 7-AGNR. The Fermi level is set to zero energy. (b) The same as (a) for edge and inner $\mathrm{C}$ atoms of 6-ZGNR.

energies, crossing $p_{y}$ and $p_{x}$ bands (except in the -3 to $3 \mathrm{eV}$ region), we have included a large number of bands (ten bands per $\mathrm{C}$ atom here) for the construction of the Wannier orbitals to make sure that enough bands are selected such that the entire correlated $p_{z}$ electron character is contained.

For entangled states the probability $c_{k m}^{\sigma}<1$ in Eq. (6), while for disentangled states $c_{k m}^{\sigma}=1$. Then, the probability of an electron to be in the $p_{z}$ correlated subspace before and after a transition $\varphi_{\boldsymbol{k} m}^{\sigma} \rightarrow \varphi_{\boldsymbol{k}^{\prime} m^{\prime}}^{\sigma}$ is given by

$$
p_{\boldsymbol{k} m \rightarrow \boldsymbol{k}^{\prime} m^{\prime}}^{\sigma}=c_{\boldsymbol{k} m}^{\sigma} c_{\boldsymbol{k}^{\prime} m^{\prime}}^{\sigma}
$$

Thus for disentangled states $p_{\boldsymbol{k} m \rightarrow \boldsymbol{k}^{\prime} m^{\prime}}^{\sigma}=1$, and for entangled states $p_{k m \rightarrow k^{\prime} m^{\prime}}^{\sigma}<1$. The polarization function $P_{z}$ now 
becomes

$$
\begin{aligned}
& P_{z}\left(\boldsymbol{r}, \boldsymbol{r}^{\prime}, \omega\right) \\
& =\sum_{\sigma} \sum_{\boldsymbol{k}, m}^{\text {occ }} \sum_{\boldsymbol{k}^{\prime}, m^{\prime}}^{\text {unocc }}\left(p_{\boldsymbol{k} m \rightarrow \boldsymbol{k}^{\prime} m^{\prime}}^{\sigma}\right) \varphi_{\boldsymbol{k} m}^{\sigma}(\boldsymbol{r}) \varphi_{\boldsymbol{k}^{\prime} m^{\prime}}^{\sigma *}(\boldsymbol{r}) \varphi_{\boldsymbol{k} m}^{\sigma *}\left(\boldsymbol{r}^{\prime}\right) \varphi_{\boldsymbol{k}^{\prime} m^{\prime}}^{\sigma}\left(\boldsymbol{r}^{\prime}\right) \\
& \quad \times\left[\frac{1}{\omega-\epsilon_{\boldsymbol{k}^{\prime} m^{\prime}}^{\sigma}+\epsilon_{\boldsymbol{k} m}^{\sigma}+i \eta}-\frac{1}{\omega+\epsilon_{\boldsymbol{k}^{\prime} m^{\prime}}^{\sigma}-\epsilon_{\boldsymbol{k} m}^{\sigma}-i \eta}\right] \cdot
\end{aligned}
$$

By calculating the total polarization from Eq. (3) and $P_{z}$ from Eq. (10), $P_{r}$ can be obtained from Eq. (4).

For completeness, the effective Coulomb matrix within the selected subspace is computed by

$$
\begin{aligned}
& U_{i n_{1}, j n_{3}, i n_{2}, j n_{4}}^{\sigma_{1}, \sigma_{2}}(\omega) \\
& \quad=\iint d \boldsymbol{r} d \boldsymbol{r}^{\prime} w_{i n_{1}}^{\sigma_{1} *}(\boldsymbol{r}) w_{j n_{3}}^{\sigma_{2} *}\left(\boldsymbol{r}^{\prime}\right) U\left(\boldsymbol{r}, \boldsymbol{r}^{\prime}, \omega\right) w_{j n_{4}}^{\sigma_{2}}\left(\boldsymbol{r}^{\prime}\right) w_{i n_{2}}^{\sigma_{1}}(\boldsymbol{r}) .
\end{aligned}
$$

In the present case, we consider only a single Wannier orbital $w_{R}(r)$ of $p_{z}$ orbital character. The local Hubbard $U$ parameter is then identical to the matrix element $\quad U(\omega)=\iint\left|w_{\mathbf{R}}(r)\right|^{2} U\left(r, r^{\prime} ; \omega\right)\left|w_{\mathbf{R}}\left(r^{\prime}\right)\right|^{2} d^{3} r d^{3} r^{\prime}$. Off-site elements are defined as $U\left(\mathbf{R}-\mathbf{R}^{\prime}, \omega\right)=$ $\iint\left|w_{\mathbf{R}}(r)\right|^{2} U\left(r, r^{\prime} ; \omega\right)\left|w_{\mathbf{R}^{\prime}}\left(r^{\prime}\right)\right|^{2} d^{3} r d^{3} r^{\prime}$. In this work we consider only the static limit $(\omega=0)$. Note that although the matrix elements of the Coulomb potential are formally spin dependent due to the spin dependence of the Wannier functions, we find that this dependence is negligible in practice.

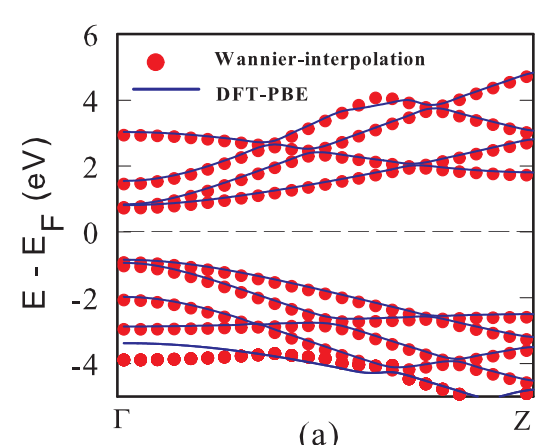

(a)

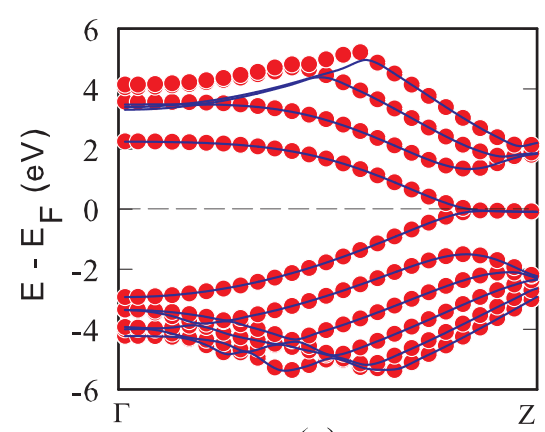

(c)

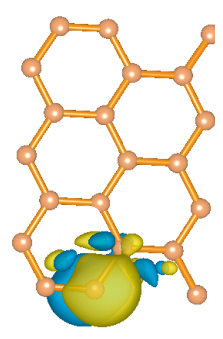

(b)

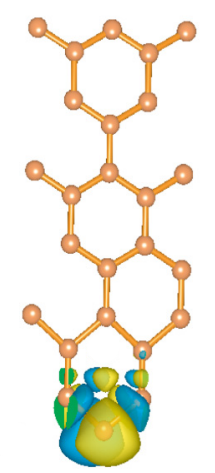

(d)
FIG. 3. (a) and (c) DFT-PBE and Wannier-interpolated band structure of 7-AGNR and 6-ZGNR, respectively. (b) and (d) The $p_{z}$-like MLWF for edge $\mathrm{C}$ atoms of these two systems.
Ground-state DFT calculations are carried out using the FLAPW method as implemented in the FLEUR code [56]. We use the generalized gradient approximation (GGA) parameterized by Perdew, Burke, and Ernzerhof (PBE) [57] for the exchange-correlation energy functional. Dense $24 \times$ $1 \times 1$ and $24 \times 24 \times 1$ k-point grids are used for unit cells of GNRs and pristine graphene, respectively. A linear momentum cutoff of $G_{\max }=4.5 \mathrm{bohr}^{-1}$ is chosen for the plane waves. The DFT calculations are used as an input for the SPEX code [58] to perform RPA and constrained RPA calculations for the fully screened and partially screened (Hubbard $U$ ) Coulomb interaction [54,55,59]. The maximally localized Wannier functions (MLWFs) are constructed with the Wannier library $[60,61]$ using ten bands per carbon atom and a dense k-point grid of $40 \times 1 \times 1$ and $18 \times 18 \times 1$ for GNRs and pristine graphene, respectively.

To verify the validity of calculated Wannier functions, in Figs. 3(a) and 3(c) we present the DFT-PBE band structure and Wannier-interpolated bands obtained from the subspace selected by projecting onto atomic $p_{z}$ orbitals on each $\mathrm{C}$ atom for 7-AGNR and 6-ZGNR, respectively. We have also visualized the $p_{z}$-like MLWFs of edge C atoms in Figs. 3(b) and 3(d) for two systems. As seen from the band structures, the overall agreement between original and Wannier-interpolated bands is quite good. Small deviations appear for states far from the Fermi energy $E_{F}$.

\section{RESULTS AND DISCUSSION}

We start with the discussion of on-site Coulomb interaction parameters for the GNRs with armchair edges having four different widths, i.e., $w_{a}=6.2 \AA$ (6-AGNR), $w_{a}=7.4 \AA$ (7AGNR), $w_{a}=8.6 \AA$ (8-AGNR), and $w_{a}=9.9 \AA$ (9-AGNR). In Fig. 4 we present on-site effective Coulomb interaction parameters (Hubbard $U$ ) as well as the fully screened interaction $W$ in order to get insight into the contribution of $p_{z}$ electrons to the total screening process. For comparison, the corresponding $U$ and $W$ values for pristine graphene are presented with dashed lines. Generally, the patterning of graphene into armchair ribbons enhances the on-site Coulomb interaction $U$ and $W$ compared to pristine graphene due to the existence of the band gap in the single-particle spectrum. For example, considering the smallest armchair system, 6-AGNR, the calculated $U(W)$ values turn out to be around $9.2 \mathrm{eV}$ $(5.5 \mathrm{eV})$ for all $\mathrm{C}$ atoms compared to that of $8.7 \mathrm{eV}(4.2 \mathrm{eV})$ for pristine graphene. The enhancement of the Coulomb interaction is stronger for the fully screened $W$, which reveals that the contribution of $p_{z} \longrightarrow p_{z}$ transitions is small in armchair GNRs, presumably because of the existence of a band gap. We find that partially (fully) screened Coulomb interaction parameters $U(W)$ for different carbon atoms across the armchair nanoribbon are nearly identical. To see the contribution of different $\mathrm{C}$ atoms, we present the projected DOS for inner and edge $\mathrm{C}$ atoms in the case of 7-AGNR. As seen in Figs. 5(d) and 5(e), the value of the band gap and the contribution of $p_{z}$ states around $E_{F}$ are similar for the two $\mathrm{C}$ atoms. This explains why the $\mathrm{C}$ atoms in armchair GNRs exhibit very similar $U$ and $W$ values. Despite very similar $U$ and $W$ within each armchair GNR, strong oscillations are seen with increasing ribbon width, especially for $W$, which 


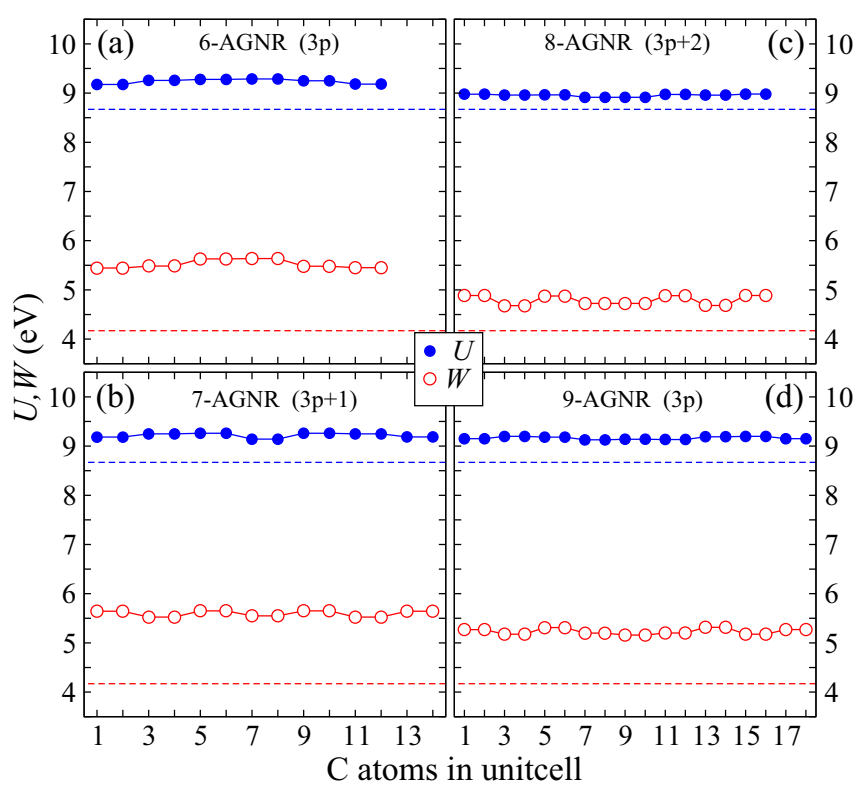

FIG. 4. Calculated partially screened on-site interaction $U$ and fully screened Coulomb interaction $W$ parameters for carbon $p_{z}$ electrons for four GNRs with armchair edges (a) 6-AGNR, (b) 7AGNR, (c) 8-AGNR, and (d) 9-AGNR. For comparison, $U$ and $W$ of pristine graphene are also included (dashed lines).

can be attributed to the quantum confinement effects, as will be discussed below.

Previous ab initio calculations for AGNRs have shown that there is a close relation between nanoribbon width and its electronic properties due to quantum confinement effects $[5,7,30,62]$. It was predicted that GNRs with hydrogenpassivated armchair edges have oscillatory band gaps as a
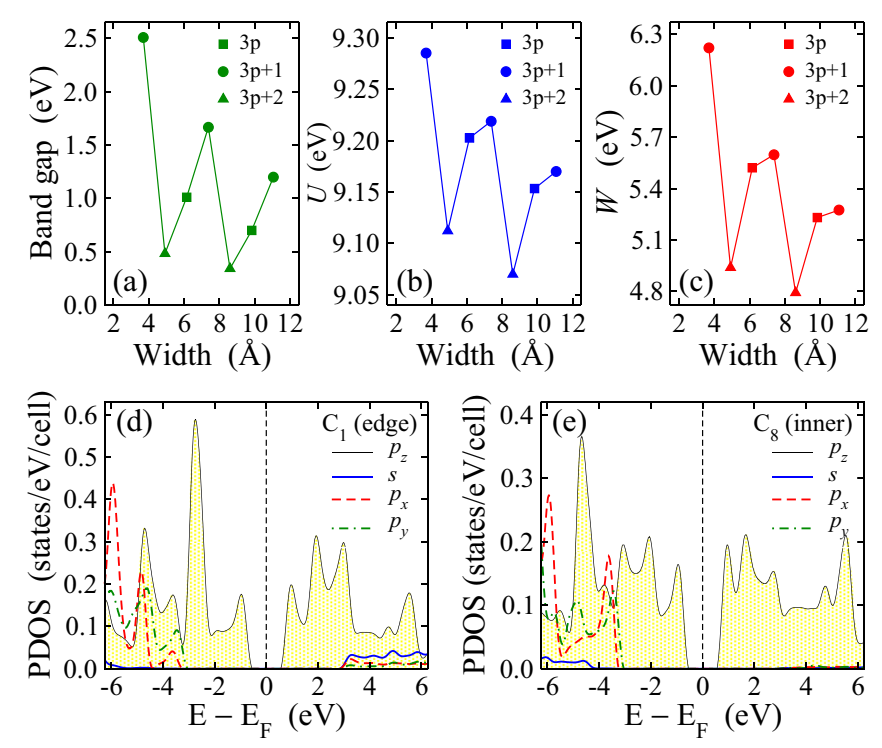

FIG. 5. (a) DFT-GGA band gaps as a function of armchair GNR width. (b) Average partially screened on-site Coulomb interaction $U$ for $p_{z}$ electrons as a function of ribbon widths of GNRs with armchair edges. (c) The same as (b) for the fully screened Coulomb interaction $W$. Projected DOS for (d) C1 and (e) C8 in 7-AGNR.
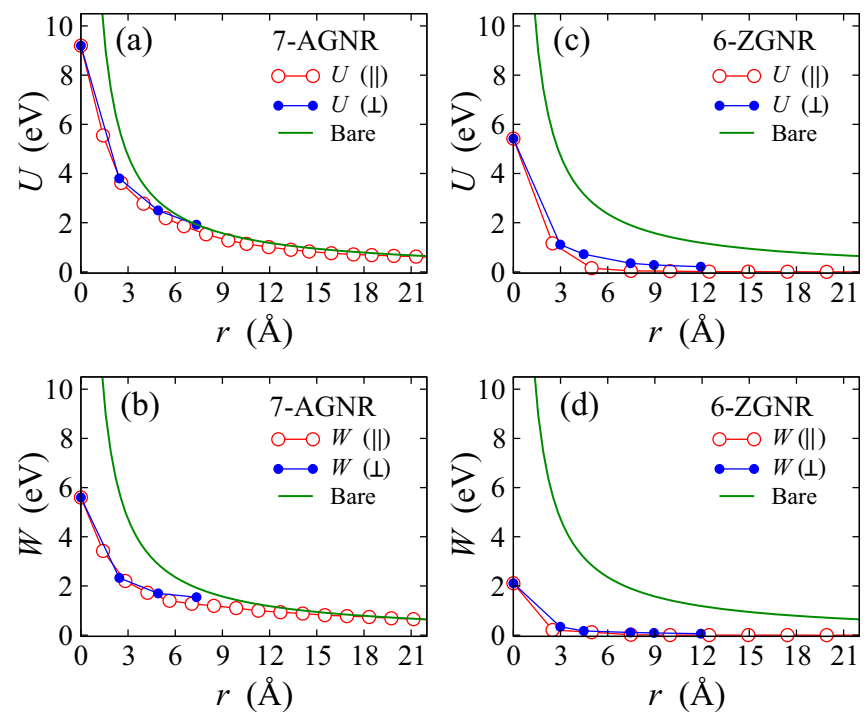

FIG. 6. (a) Partially screened Coulomb interaction $U$ for $\mathrm{C} p_{z}$ electrons as a function of distance $r$ for an armchair GNR (7-AGNR). Here, $U(\|)$ and $U(\perp)$ correspond to interactions along the ribbon and across the ribbon, respectively. For comparison the bare interaction is presented. (b) The same as (a) for the fully screened Coulomb interaction $W$. (c) and (d) The same as (a) and (b) for zigzag GNR (6-ZGNR).

function of the ribbon width $[7,30]$. Theoretical predictions of this oscillatory behavior of the band gap in AGNRs were recently confirmed by experiments $[5,6]$. To reveal the influence of quantum confinement effects on the screening of Coulomb interaction, we present in Fig. 5 the GGA band gap and the average $U$ and $W$ parameters of $\mathrm{C}$ atoms as a function of the ribbon width for armchair systems. Since the Coulomb matrix elements for different $\mathrm{C}$ atoms across the ribbons are nearly identical, we assign the average of Coulomb interaction to each ribbon. Quantum confinement effects give rise to oscillatory band gaps, which is reflected in the equally oscillatory behavior of the $U$ and $W$ parameters. Moreover, both partially and fully screened Coulomb interactions $(U$, $W$ ) decrease as a function of increasing ribbon widths in each family, which is in good agreement with the behavior of our calculated band gaps. The large Coulomb matrix elements for small-width armchair GNRs are in agreement with significant many-body GW corrections to the band gaps, which are in the range of $0.5-3 \mathrm{eV}$ in armchair GNRs of 0.4-1.6-nm width (see Ref. [30]). For sufficiently large widths, one expects the fully screened interaction to reach the graphene limit of $4.2 \mathrm{eV}$.

The long-range Coulomb interactions play an important role in determining the phase diagram of hexagonal carbonbased materials $[44,45,48]$. Previous ab initio studies of the effective Coulomb interaction in pristine graphene revealed a significant nonlocal part of $U$ [48]. For the present systems we expect even stronger nonlocal interactions due to reduced dimensionality and the existence of the band gap. In Fig. 6 the nonlocal partially (fully) screened Coulomb interaction parameters along the ribbon $U(\|)[W(\|)]$ and across the ribbon $U(\perp)[W(\perp)]$ are presented for 7-AGNR as a function of distance $r$ between two $\mathrm{C}$ atoms. For comparison, the corresponding nonlocal bare Coulomb interaction parameters are 

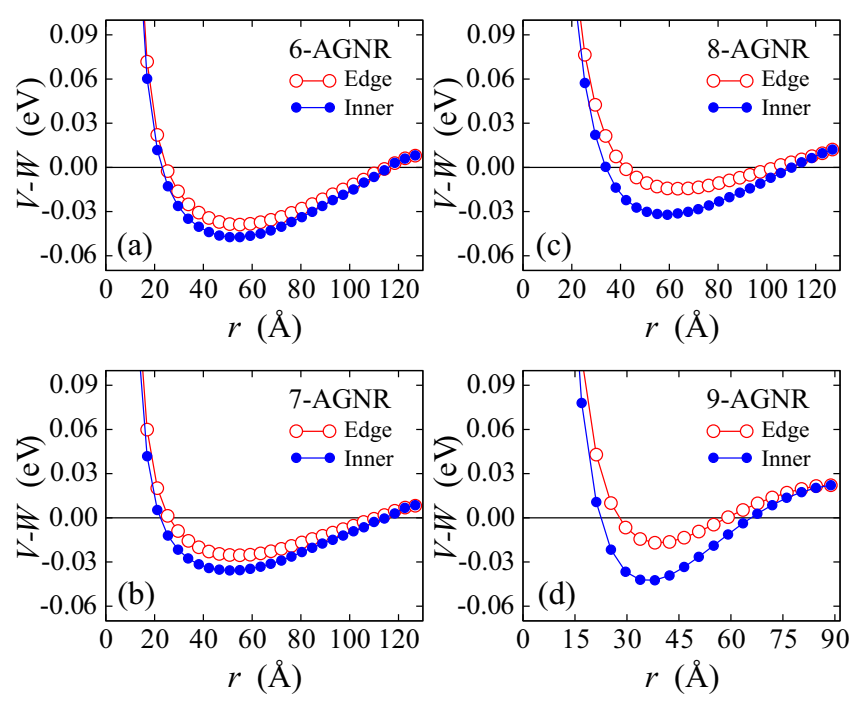

FIG. 7. The difference $(V-W)$ between bare interaction $V$ and fully screened interaction $W$ as a function of distance $r$ between two $\mathrm{C}$ atoms along the ribbon for the edge and central atoms of (a) 6AGNR, (b) 7-AGNR, (c) 8-AGNR, and (d) 9-AGNR.

also presented. We find sizable off-site $U$ and $W$ parameters at short distances which decay slowly due to the weak screening. Furthermore, the nonlocal Coulomb interaction across the ribbon is slightly larger than the one along the ribbon. The difference $V-W$ decreases with increasing distance because, at large distances, the screened interaction has to approach $1 / \varepsilon r$ and the dielectric constant $\varepsilon=1$ for low-dimensional systems. At intermediate distances, however, we see that $V-W$ can become negative, indicating a nonconventional screening of interactions in these quasi-one-dimensional armchair GNRs.

As we have seen above, the $V-W$ difference can become negative at intermediate distances for AGNRs, which is a sign of the nonconventional screening as it is observed in several low-dimensional semiconductors and insulators [32,33]. To reveal the intermediate- and long-range behavior of the Coulomb interaction in armchair GNRs we have extended the calculations of $W$ to much larger distances, and in Fig. 7 we present the $V-W$ difference for both central and edge atoms of armchair GNRs. At short distances the $V-W$ difference is positive, i.e., $W<V$ as expected, while with increasing distance the $V-W$ difference changes sign and becomes negative, which means that the screened interaction $W$ becomes larger than the bare interaction $V$. This is called antiscreening. Considering the central atoms in the smallest nanoribbon, 6-AGNR, the antiscreening is observed between the critical distances $r_{c 1}=22 \AA$, where the transition from screening to antiscreening takes place, and $r_{c 2}=115 \AA$, where the transition from antiscreening to unscreening (or very weak screening) occurs. As for 7-AGNR, 8-AGNR, and 9-AGNR, the critical distance $r_{c 1}$ is, respectively, 22, 35, and $25 \AA$ for central atoms. As expected, the antiscreening region $r_{c 2}-r_{c 1}$ decreases with increasing the ribbon width in each family of armchair systems. For example, the antiscreening tends to be more reduced in 9-AGNR than in 6-AGNR. Moreover, the antiscreening weakens as one moves from the central to the edge atoms in armchair GNRs, especially in the case of 8AGNR and 9-AGNR. The physical origin of the antiscreening in low-dimensional materials has been addressed in detail in recent publications by the present authors and will not be discussed here $[63,64]$. In general, the antiscreening in armchair GNRs is one order of magnitude smaller than the one observed in transition-metal oxide clusters $\mathrm{Fe}_{x} \mathrm{O}_{y}$. Furthermore, the critical distance for the onset of antiscreening in armchair GNRs is significantly longer than in zero-dimensional molecules and clusters (benzyne, $r_{c 1}=3-4 \AA$ [32]; $\mathrm{Fe}_{x} \mathrm{O}_{y}$, $r_{c 1}=2-3 \AA$ [63]; and $\mathrm{Nb}_{4} \mathrm{Co}, r_{c 1}=3.9 \AA$ [64]). On the other hand, the onset of antiscreening in armchair GNRs is comparable to that of the quasi-one-dimensional singlewall carbon nanotubes, for which $r_{c 1}$ is found to be around $20 \AA$ [33].

So far we have seen that a distinct feature of electrons in armchair GNRs is the significantly reduced and nonconventional dielectric screening of Coulomb interactions. An important consequence of this nonconventional screening is the formation of tightly bound excitons with binding energies of about $1.5 \mathrm{eV}$, as observed experimentally in armchair GNRs $[39,41]$. This is one order of magnitude larger than the corresponding binding energy in bulk semiconductors and is in good agreement with ab initio calculations [31,36,37,65]. It is worth noting that other low-dimensional semiconductors and insulators such as graphane [34], fluorographene [66], and transition-metal dichalcogenides [67-72] possesses tightly bound excitons, and their dispersion strongly deviates from the hydrogenic Rydberg series, revealing a significantly reduced and nonlocal dielectric screening of the Coulomb interaction in these materials.

Above we discussed the screening of the Coulomb interaction in armchair GNRs. In the following we will consider zigzag GNRs of different widths $\left[w_{z}=12 \AA\right.$ (6-ZGNR), $w_{z}=14 \AA$ (7-ZGNR), and $w_{z}=16 \AA$ (8-ZGNR)]. GNRs with zigzag edges possess magnetic edge states, but our calculations have shown that the spin polarization has a negligible influence $(0.1-0.3 \mathrm{eV})$ on the Coulomb interaction parameters, and thus the on-site $U$ and $W$ values presented in Fig. 8 are for the non-spin-polarized case. As can be seen, the fully screened Coulomb interaction parameters for the inner carbon atoms are very similar to the ones for pristine graphene, while for the edge atoms we obtain a substantial reduction in $W$. A similar difference between the parameters of edge and inner atoms is observed for the Hubbard $U$ parameter, but $U$ is considerably smaller than in pristine graphene. The strong reduction in $U$ and $W$ for edge atoms can be explained by the atom- and orbital-resolved DOSs presented in Fig. 8. The $p_{z}$ electrons for the edge atoms are responsible for this reduction. As seen in Fig. 8, the edge atom has a large $p_{z}$ peak around $E_{F}$, while for inner atoms this peak almost disappears. As a consequence, the contribution of the $p_{z} \rightarrow \sigma$ channel (as well as $p_{z} \rightarrow p_{z}$ ) to the electronic polarization function increases substantially, which increases the dielectric screening and as a result gives rise to smaller $U(W)$ Coulomb interaction parameters for the edge atoms. Moreover, edge states in zigzag GNRs strongly influence nonlocal Coulomb interactions, making them extremely short range. As shown in Figs. 6(c) and 6(d), the Hubbard $U$ and $W$ along the ribbon are fully screened at distances larger 

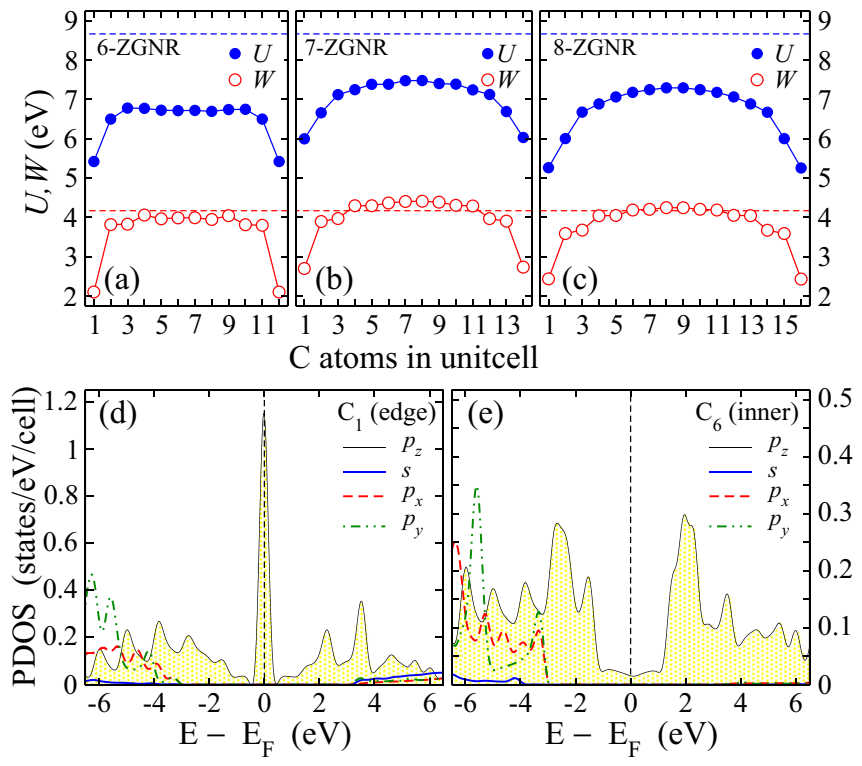

FIG. 8. The same as Fig. 5 for (a) 6-ZGNR, (b) 7-ZGNR, and (c) 8-ZGNR. The projected non-spin-polarized DOS for (d) C1 and (e) C6 in 6-ZGNR.

than $5 \AA$, while across the ribbon $U$ becomes slightly long range $(\sim 10 \AA)$.

The gradient of the long-range Coulomb interaction $U(r)$ plays a crucial role in determining the strength of electronic correlations and classification of the materials $[32,46]$. In this respect, due to long-range Coulomb interactions the armchair GNRs can be considered weakly correlated materials, and thus standard DFT is expected to describe appropriately the electronic properties of these materials. On the other hand, due to the large gradient of the Coulomb interaction zigzag GNRs can be seen as correlated systems exhibiting rich physical behavior, such as edge ferromagnetism, as will be briefly discussed below. It is worth noting that according to the Hubbard model, strength of the correlation is determined by the ratio of on-site Coulomb interaction $U$ and the bandwidth $W_{b}\left(U / W_{b}\right)$. When $U / W_{b}>1$, a material is classified as a strongly correlated system. From DFT electronic structure, the obtained $W_{b}$ for $p_{z}$ bands of edge atoms of ZGNRs vary in the range $4.0-5.0 \mathrm{eV}$. Our calculated $U$ parameters presented in Fig. 8 range between 5.0 and $6.0 \mathrm{eV}$, and thus $U / W_{b} \sim 1$,

TABLE I. Nearest-neighbor $\left(t_{01}\right)$, next-nearest-neighbor $\left(t_{02}, t_{02}^{\prime}\right)$, and third-nearest-neighbor $\left(t_{03}\right)$ hopping parameters for edge atom $\mathrm{C}_{1}$ and inner atom $\mathrm{C}_{7}$ in 6-ZGNR. Here, $t_{02}^{\prime}$ is the next-nearestneighbor hopping parameter along the ribbon. Distance between two $\mathrm{C}$ atoms $d_{0 n}$ is also reported.

\begin{tabular}{lclcc}
\hline \hline \multicolumn{2}{c}{ Edge atom $\mathrm{C}_{1}$} & & \multicolumn{2}{c}{ Inner atom $\mathrm{C}_{7}$} \\
\cline { 1 - 2 } \cline { 5 - 6 } Distance & Hopping & & Distance & Hopping \\
\hline$d_{01}=1.43 \AA$ & $t_{01}=-2.68 \mathrm{eV}$ & & $d_{01}=1.47 \AA$ & $t_{01}=-2.59 \mathrm{eV}$ \\
$d_{02}=2.49 \AA$ & $t_{02}=-0.29 \mathrm{eV}$ & & $d_{02}=2.51 \AA$ & $t_{02}=-0.24 \mathrm{eV}$ \\
$d_{03}=2.87 \AA$ & $t_{03}=-0.18 \mathrm{eV}$ & & $d_{03}=2.91 \AA$ & $t_{03}=-0.21 \mathrm{eV}$ \\
$d_{02}^{\prime}=1.41 \AA$ & $t_{02}^{\prime}-0.33 \mathrm{eV}$ & & $d_{02}^{\prime}=1.45 \AA$ & $t_{02}^{\prime}=-0.28 \mathrm{eV}$ \\
\hline \hline
\end{tabular}
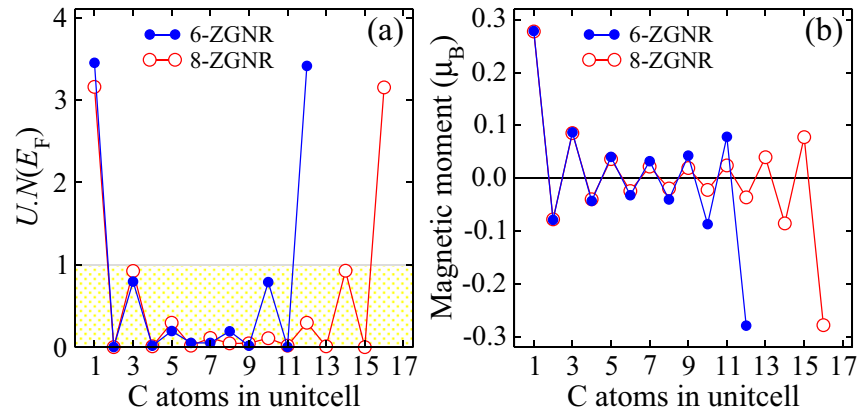

FIG. 9. (a) Stoner criterion corresponding to two ZGNR systems, 6-ZGNR and 8-ZGNR. (b) Calculated magnetic moments (in units of $\mu_{B}$ ) of different carbon atoms for these two systems (see Fig. 1).

which means that ZGNRs are moderately correlated materials. For construction of the ab initio model Hamiltonians, in addition to Coulomb interaction parameters, single-particle energy spectra, i.e., hopping parameters, are needed. The latter is presented in Table I for the case of 6-ZGNR from the firstto third-nearest neighbors obtained from Wannier functions. The results are in good agreement with reported values of $t_{01}, t_{02}$, and $t_{03}$, which are in the range of 2.5-3, 0.1-0.5, and $0.3-0.5 \mathrm{eV}$, respectively [73-78].

Finally, we discuss the appearance of ferromagnetism in GNRs with zigzag edges. Due to the peculiar geometric structure, the $p_{z}$ orbitals of the edge carbon atoms yield the formation of nonbonding $\pi$ orbitals, which makes these materials metallic [see Fig. 8(d)] in non-spin-polarized calculations, and thus the system becomes unstable against the formation of a magnetic ground state if the Stoner criterion is satisfied. It is worth noting that due to the itinerant character of the $p_{z}$ electrons, the Stoner model is well suited for the zigzag GNRs. In Fig. 9 we present the Stoner criterion for zigzag GNRs with two different widths (6-ZGNR and 8-ZGNR). As seen in both cases, only the edge atoms satisfy the criterion, and indeed in spin-polarized calculations we obtain a sizable magnetic moment $\left(0.29 \mu_{B}\right)$ for the edge atoms. Small magnetic moments in inner atoms are induced. The edge magnetic moments are coupled antiferromagnetically across the ribbon, while the coupling is ferromagnetic along the ribbon. Recent scanning tunnel microscopy experiments have shown that large spin splittings at the Fermi level are present in zigzag GNRs narrower than a couple of nanometers [5,79], providing evidence for a room-temperature edge ferromagnetism in these materials. Using experimental results, the magnitude of the on-site repulsion $U$ was estimated to be around $4.32 \mathrm{eV}$ for nanoribbons with an ideal zigzag edge [5]. The estimated value is in reasonable agreement with our calculated $U$ of $5 \mathrm{eV}$.

\section{CONCLUSION}

Employing first-principles calculations in conjunction with the random-phase approximation we have studied the screening of the long-range Coulomb interactions for $p_{z}$ electrons in armchair and zigzag GNRs as a function of the ribbon width. Our calculations have shown that in GNRs with armchair edges quantum confinement effects lead to oscillatory 
behavior of the on-site screened Coulomb interaction with ribbon width. Furthermore, the reduced dimensionality and the existence of a band gap results in a nonconventional screening of the Coulomb interaction; that is, at intermediate distances $(20 \AA<r<100 \AA$ ) we obtain an antiscreening (i.e., the screened interaction $W$ becomes larger than the bare interaction $V$ ). In the case of GNRs with zigzag edges, the situation is quite different; the presence of edge states strongly affects the screening, which leads to a strong reduction of the effective Coulomb interaction (Hubbard $U$ ) parameter at the edge. We find that the interactions turn out to be local; the nonlocal part is strongly screened due to edge states, making GNRs with zigzag edges correlated systems, as the gradient of the Coulomb interaction is much larger than that of the GNRs with armchair edges. On the basis of calculated effective Coulomb interaction parameters $U$ we discuss the appearance of ferromagnetism at zigzag edges of GNRs using the Stoner model. The calculated $U$ parameters are not only important for a fundamental understanding of ferromagnetism in zigzag GNRs, but they can also serve as effective interaction parameters to be used in model Hamiltonians applied to describe electronic, optical, and magnetic properties. This provides model parameters from first principles rather than having to fit them to experimental data, thus increasing the predictive power of model Hamiltonian calculations.

\section{ACKNOWLEDGMENTS}

E.S. and I.M. gratefully acknowledge the funding provided by the European Union (EFRE). This work has been supported by the Iran Science Elites Federation through Grant No. 2017. H.H. and F.B. acknowledge the computational resources provided by the Physics Department of the University of Guilan.
[1] A. K. Geim and K. S. Novoselov, Nat. Mater. 6, 183 (2007).

[2] M. I. Katsnelson, Mater. Today 10, 20 (2007).

[3] Exploring graphene-Recent research advances, edited by S. Das Sarma, A. K. Geim, P. Kim, and A. H. MacDonald, special issue of Solid State Communications, Vol. 143 (2007), pp. 1-126.

[4] S. S. Rao, S. N. Jammalamadaka, A. Stesmans, V. V. Moshchalkov, J. v. Tol, D. V. Kosynkin, A. HigginbothamDuque, and J. M. Tour, Nano Lett. 12, 1210 (2012).

[5] G. Zsolt Magda, X. Jin, I. Hagymási, P. Vancsó, Z. Osváth, P. Nemes-Incze, C. Hwang, L. P. Biró, and L. Tapasztó, Nature (London) 514, 608 (2014).

[6] W. X. Wang, M. Zhou, X. Li, S. Y. Li, X. Wu, W. Duan, and L. He, Phys. Rev. B 93, 241403(R) (2016).

[7] Y.-W. Son, M. L. Cohen, and S. G. Louie, Phys. Rev. Lett. 97, 216803 (2006).

[8] H. Lee, Y.-W. Son, N. Park, S. Han, and J. Yu, Phys. Rev. B 72, 174431 (2005).

[9] K. Nakada, M. Fujita, G. Dresselhaus, and M. S. Dresselhaus, Phys. Rev. B 54, 17954 (1996).

[10] H. Feldner, Z. Y. Meng, T. C. Lang, F. F. Assaad, S. Wessel, and A. Honecker, Phys. Rev. Lett. 106, 226401 (2011).

[11] Y. Niimi, T. Matsui, H. Kambara, K. Tagami, M. Tsukada, and H. Fukuyama, Phys. Rev. B 73, 085421 (2006).

[12] X. Li, X. Wang, L. Zhang, S. Lee, and H. Dai, Science 319, 1229 (2008).

[13] C. Tao, L. Jiao, O. V. Yazyev, Y.-C. Chen, J. Feng, X. Zhang, R. B. Capaz, J. M. Tour, A. Zettl, S. G. Louie, H. Dai, and M. F. Crommie, Nat. Phys. 7, 616 (2011).

[14] M. Y. Han, B. Özyilmaz, Y. Zhang, and P. Kim, Phys. Rev. Lett. 98, 206805 (2007).

[15] X. Wang, Y. Ouyang, X. Li, H. Wang, J. Guo, and H. Dai, Phys. Rev. Lett. 100, 206803 (2008).

[16] Y.-W. Son, M. L. Cohen, and S. G. Louie, Nature (London) 444, 347 (2006).

[17] L. Brey, H. A. Fertig, and S. Das Sarma, Phys. Rev. Lett. 99, 116802 (2007).

[18] M. Wimmer, İ. Adagideli, S. Berber, D. Tománek, and K. Richter, Phys. Rev. Lett. 100, 177207 (2008).
[19] N. Tombros, C. Jozsa, M. Popinciuc, H. T. Jonkman, and B. J. van Wees, Nature (London) 448, 571 (2007).

[20] E. Kan, Z. Li, J. Yang, and J. G. Hou, J. Am. Chem. Soc. 130, 4224 (2008).

[21] Z. H. Chen, Y. M. Lin, M. J. Rooks, and P. Avouris, Physica E (Amsterdam, Neth.) 40, 228 (2007).

[22] L. Tapaszto, G. Dobrik, P. Lambin, and L. P. Biro, Nat. Nanotechnol. 3, 397 (2008).

[23] L. Y. Jiao, L. Zhang, X. R. Wang, G. Diankov, and H. J. Dai, Nature (London) 458, 877 (2009).

[24] D. V. Kosynkin, A. L. Higginbotham, A. Sinitskii, J. R. Lomeda, A. Dimiev, B. K. Price, and J. M. Tour, Nature (London) 458, 872 (2009).

[25] A. L. Elias, A. R. Botello-Mendez, D. Meneses-Rodriguez, V. J. Gonzalez, D. Ramirez-Gonzalez, L. Ci, E. Munoz-Sandoval, P. M. Ajayan, H. Terrones, and M. Terrones, Nano Lett. 10, 366 (2009).

[26] L. Jiao, X. Wang, G. Diankov, H. Wang, and H. Dai, Nat. Nanotechnol. 5, 321 (2010).

[27] S. S. Datta, D. R. Strachan, S. M. Khamis, and A. T. C. Johnson, Nano Lett. 8, 1912 (2008).

[28] J. Campos-Delgado, J. M. Romo-Herrera, X. Jia, D. A. Cullen, H. Muramatsu, Y. A. Kim, T. Hayashi, Z. Ren, D. J. Smith, Y. Okuno, T. Ohba, H. Kanoh, K. Kaneko, M. Endo, H. Terrones, M. S. Dresselhaus, and M. Terrones, Nano Lett. 8, 2773 (2008).

[29] L. C. Campos, V. R. Manfrinato, J. D. Sanchez-Yamagishi, J. Kong, and P. Jarillo-Herrero, Nano Lett. 9, 2600 (2009).

[30] L. Yang, C. H. Park, Y. W. Son, M. L. Cohen, and S. G. Louie, Phys. Rev. Lett. 99, 186801 (2007).

[31] L. Yang, M. L. Cohen, and S. G. Louie, Nano Lett. 7, 3112 (2007).

[32] J. van den Brink and G. A. Sawatzky, Europhys. Lett. 50, 447 (2000).

[33] J. Deslippe, M. Dipoppa, D. Prendergast, M. V. O. Moutinho, R. B. Capaz, and S. G. Louie, Nano Lett. 9, 1330 (2009).

[34] P. Cudazzo, I. V. Tokatly, and A. Rubio, Phys. Rev. B 84, 085406 (2011). 
[35] M. van Schilfgaarde and M. I. Katsnelson, Phys. Rev. B 83, 081409(R) (2011).

[36] D. Prezzi, D. Varsano, A. Ruini, A. Marini, and E. Molinari, Phys. Rev. B 77, 041404(R) (2008).

[37] D. Prezzi, D. Varsano, A. Ruini, and E. Molinari, Phys. Rev. B 84, 041401 (2011).

[38] S. Wang and J. Wang, J. Phys. Chem. C 116, 10193 (2012).

[39] R. Denk, M. Hohage, P. Zeppenfeld, J. Cai, C. A. Pignedoli, H. Sode, R. Fasel, X. Feng, K. Mullen, S. Wang, D. Prezzi, A. Ferretti, A. Ruini, E. Molinari, and P. Ruffieux, Nat. Commun. 5, 4253 (2014).

[40] C. Bronner, D. Gerbert, A. Broska, and P. Tegeder, J. Phys. Chem. C 120, 26168 (2016).

[41] G. Soavi, S. D. Conte, C. Manzoni, D. Viola, A. Narita, Y. Hu, X. Feng, U. Hohenester, E. Molinari, D. Prezzi, K. Mullen, and G. Cerullo, Nat. Commun. 7, 11010 (2016).

[42] S. Sorella and E. Tosatti, Europhys. Lett. 19, 699 (1992).

[43] T. Paiva, R. T. Scalettar, W. Zheng, R. R. P. Singh, and J. Oitmaa, Phys. Rev. B 72, 085123 (2005).

[44] I. F. Herbut, Phys. Rev. Lett. 97, 146401 (2006).

[45] C. Honerkamp, Phys. Rev. Lett. 100, 146404 (2008).

[46] M. Schuler, M. Rosner, T. O. Wehling, A. I. Lichtenstein, and M. I. Katsnelson, Phys. Rev. Lett. 111, 036601 (2013).

[47] S. Chacko, D. Nafday, D. G. Kanhere, and T. Saha-Dasgupta, Phys. Rev. B 90, 155433 (2014).

[48] T. O. Wehling, E. Şaşıŏlu, C. Friedrich, A. I. Lichtenstein, M. I. Katsnelson, and S. Blügel, Phys. Rev. Lett. 106, 236805 (2011).

[49] E. Şaşığlu, H. Hadipour, C. Friedrich, S. Blügel, and I. Mertig, Phys. Rev. B 95, 060408(R) (2017).

[50] N. D. Mermin and H. Wagner, Phys. Rev. Lett. 17, 1133 (1966).

[51] O. V. Yazyev and M. I. Katsnelson, Phys. Rev. Lett. 100, 047209 (2008).

[52] O. V. Yazyev, Rep. Prog. Phys. 73, 056501 (2010).

[53] K. Wakabayashi, K.-I. Sasaki, T. Nakanishi, and T. Enoki, Sci. Technol. Adv. Mater. 11, 054504 (2010).

[54] F. Aryasetiawan, M. Imada, A. Georges, G. Kotliar, S. Biermann, and A. I. Lichtenstein, Phys. Rev. B 70, 195104 (2004); F. Aryasetiawan, K. Karlsson, O. Jepsen, and U. Schönberger, ibid. 74, 125106 (2006); T. Miyake, F. Aryasetiawan, and M. Imada, ibid. 80, 155134 (2009).

[55] E. Şaşığlu, C. Friedrich, and S. Blügel, Phys. Rev. B 83, 121101(R) (2011); Phys. Rev. Lett. 109, 146401 (2012).

[56] FLEUR, http://www.flapw.de.

[57] J. P. Perdew, K. Burke, and M. Ernzerhof, Phys. Rev. Lett. 77, 3865 (1996).
[58] C. Friedrich, S. Blügel, and A. Schindlmayr, Phys. Rev. B 81, 125102 (2010).

[59] Y. Nomura, M. Kaltak, K. Nakamura, C. Taranto, S. Sakai, A. Toschi, R. Arita, K. Held, G. Kresse, and M. Imada, Phys. Rev. B 86, 085117 (2012); B-C. Shih, Y. Zhang, W. Zhang, and P. Zhang, ibid. 85, 045132 (2012).

[60] A. A. Mostofi, J. R. Yates, Y.-S. Lee, I. Souza, D. Vanderbilt, and N. Marzari, Comput. Phys. Commun. 178, 685 (2008).

[61] F. Freimuth, Y. Mokrousov, D. Wortmann, S. Heinze, and S. Blügel, Phys. Rev. B 78, 035120 (2008).

[62] V. Barone, O. Hod, and G. E. Scuseria, Nano Lett. 6, 2748 (2006).

[63] L. Peters, E. Şaşığlu, S. Rossen, C. Friedrich, S. Blügel, and M. I. Katsnelson, Phys. Rev. B 95, 155119 (2017).

[64] L. Peters, E. Şaşığlu, I. Mertig, and M. I. Katsnelson, Phys. Rev. B 97, 045121 (2018).

[65] X. Zhu and H. Su, J. Phys. Chem. A 115, 11998 (2011).

[66] F. Karlicky and M. Otyepka, J. Chem. Theory. Comput. 9, 4155 (2013).

[67] A. Chernikov, T. C. Berkelbach, H. M. Hill, A. Rigosi, Y. Li, O. B. Aslan, D. R. Reichman, M. S. Hybertsen, and T. F. Heinz, Phys. Rev. Lett. 113, 076802 (2014).

[68] K. He, N. Kumar, L. Zhao, Z. Wang, K. F. Mak, H. Zhao, and J. Shan, Phys. Rev. Lett. 113, 026803 (2014).

[69] M. M. Ugeda, A. J. Bradley, S.-F. Shi, F. H. da Jornada, Y. Zhang, D. Y. Qiu, W. Ruan, S.-K. Mo, Z. Hussain, Z.-X. Shen, F. Wang, S. G. Louie, and M. F. Crommie, Nat. Mater. 13, 1091 (2014).

[70] H. Shi, H. Pan, Y.-W. Zhang, and B. I. Yakobson, Phys. Rev. B 87, 155304 (2013).

[71] D. Y. Qiu, F. H. da Jornada, and S. G. Louie, Phys. Rev. Lett. 111, 216805 (2013).

[72] G. Berghäuser and E. Malic, Phys. Rev. B 89, 125309 (2014).

[73] J. L. McChesney, A. Bostwick, T. Ohta, T. Seyller, K. Horn, J. Gonzalez, and E. Rotenberg, Phys. Rev. Lett. 104, 136803 (2010).

[74] S. Reich, J. Maultzsch, C. Thomsen, and P. Ordejon, Phys. Rev. B 66, 035412 (2002).

[75] C. Bena and L. Simon, Phys. Rev. B 83, 115404 (2011).

[76] D. Gunlycke and C. T. White, Phys. Rev. B 77, 115116 (2008).

[77] Y. Hancock, A. Uppstu, K. Saloriutta, A. Harju, and M. J. Puska, Phys. Rev. B 81, 245402 (2010).

[78] V.-T. Tran, J. Saint-Martin, P. Dollfus, and S. Volz, AIP Adv. 7, 075212 (2017).

[79] Y. Y. Li, M. X. Chen, M. Weinert, and L. Li, Nat. Commun. 5, 4311 (2014). 